Este é um artigo publicado em acesso aberto sob uma licença Creative Commons https://creativecommons.org/licenses/by-nc/4.0/

\title{
Construindo uma universidade sustentável: uma discussão baseada no caso de uma universidade portuguesa
}

\author{
Marcelo Ximenes Aguiar Bizerril ${ }^{1}$ \\ Maria João Rosa ${ }^{2}$ \\ Teresa Carvalho ${ }^{3}$
}

Resumo: A partir do caso de uma universidade portuguesa, discutimos visões sobre o que deve ser uma Universidade Sustentável (US), bem como sobre as diferentes etapas necessárias à sua implementação. A discussão foi construída a partir da revisão da literatura sobre a sustentabilidade no ensino superior, análises de documentos da instituição, observação do campus e entrevistas com diferentes atores associados às questões relativas à sustentabilidade na universidade. A universidade estudada atua nas dimensões da sustentabilidade sugeridas na literatura, mas percebe-se que o processo de institucionalização de uma efetiva US encontra-se ainda em fase inicial. A partir do caso estudado são sugeridas ações estratégicas para as universidades que buscam a transição para US que incluem a adoção de uma perspectiva holística de US por meio da discussão e de documentos oficiais, a prática da gestão democrática e participativa, e a criação de canais formais de diálogo com a sociedade.

Palavras-chave: Sustentabilidade. Universidade Sustentável. Gestão da Educação Superior.

\section{Building a sustainable university: a discussion based on a portuguese university case}

Abstract: Based on the case of a Portuguese university, we discuss views about what should be a Sustainable University (SU) and the processes to institutionalise it. The discussion is built from a literature review on sustainability in higher education, the analysis of institutional documents, campus observation and interviews with different individuals associated with sustainability issues at the university. The studied university acts in the dimensions of sustainability suggested in the literature, but it is clear that the institutionalization process of an effective SU is still in its early phases. Starting from the studied case we suggest strategic actions for universities that seek the transition to SU, which include the adoption of a holistic perspective of SU through discussion and the issue of official documents, the practice of democratic and participatory management, and the establishment of formal means of dialogue with society.

Key words: Sustainability. Sustainable University. Higher Education Governance. 


\title{
1 A sustentabilidade e as Universidades sustentáveis
}

Nas últimas duas décadas tem se acentuado o reconhecimento do papel que as universidades podem desempenhar no apoio à transformação das sociedades em sustentáveis. Dentre as ações possíveis destaca-se a institucionalização da sustentabilidade no ensino superior com vistas à transição para um modelo que tem sido chamado de Universidade Sustentável (US).

Velazquez, Munguia, Platt e Taddei (2006, p. 811) apresentam a seguinte definição para a US:

\begin{abstract}
Uma instituição de ensino superior, como um todo ou em parte, que busque a promoção, a nível regional ou global, da minimização de impactos negativos ambientais, sociais, econômicos e à saúde gerados pelo uso dos seus recursos quando do cumprimento de suas funções de ensino, pesquisa, extensão e manutenção de forma a ajudar a sociedade a fazer a transição para estilos de vida sustentáveis.
\end{abstract}

Na visão de Disterheft, Caeiro, Ramos e Azeiteiro (2012), um campus sustentável deve associar os aspectos operacionais do ensino, da pesquisa e da gestão institucional - inclusive de recursos e resíduos - com a educação para a sustentabilidade, de forma que tanto a comunidade interna quanto externa venham a reconhecer e praticar estilos de vida que promovam o bemestar da atual e das futuras gerações.

A Declaração de Taillores é uma das cartas de compromisso das universidades em relação à sustentabilidade com maior repercussão global. Nela não há uma definição de US, mas um destaque da necessidade de estabelecer uma cultura de sustentabilidade e formar cidadãos ambientalmente responsáveis, a partir da prática da sustentabilidade no dia-a-dia do campus, na pesquisa, na educação e na relação com a sociedade. González-Gaudiano, MeiraCartea e Martínez-Fernández (2015), reforçam essa ideia afirmando que a gestão do espaço, dos resíduos e da energia, por meio de planejamentos mais ecológicos, têm pouco resultado se não houver a participação consciente dos distintos grupos (professores, funcionários, estudantes) que integram a comunidade universitária.

Considerando o conjunto teórico desenvolvido na última década (veja, por exemplo, CORTESE, 2003; LOZANO, 2006; VELAZQUEZ; MUNGUIA; PLATT; TADDEI, 2006; ALSHUWAIKHAT; ABUBAKAR， 2008; KARATZOGLOU， 2013; LOZANO; CELEUMANS; ALONSO-ALMEIDA; HUISINGH; LOZANO, F.; WAAS; LAMBRECHTS; LUKMAN; HUGÉ, 2015; DISTERHEFT; CAEIRO; AZEITEIRO; LEAL FILHO, 2014; AMARAL; MARTINS; GOUVEIA, 2015), pode-se concluir que é esperado que a US tenha atuação consistente e consciente nas seguintes sete dimensões: educação, investigação, gestão 
do campus, extensão, avaliação e relato, diretrizes institucionais, e vivências de sustentabilidade no campus (veja maior detalhamento das dimensões em LOZANO; CELEUMANS; ALONSOALMEIDA; HUISINGH; LOZANO, F.; WAAS; LAMBRECHTS; LUKMAN; HUGÉ, 2015).

Contudo, verifica-se que um conjunto significativo de tentativas de implementação da US trazem uma forte referência ao conceito de desenvolvimento sustentável, baseado no tripé social-econômico-ambiental. Vale ressaltar que esse modelo é alvo de críticas, tanto por desconsiderar aspectos importantes como, por exemplo, as dimensões cultural e política da sustentabilidade (BURFORD; HOOVER; VELASCO; JANOUŠKOVÁ; JIMENEZ; PIGGOT; PODGER; HARDER, 2013), como por preservar alguns valores e princípios de um sistema econômico responsável pela desigualdade e crise ecológica planetária (GONZÁLEZGAUDIANO; SILVA-RIVERA, 2015). De fato, a atual situação de alarme socioambiental deve-se diretamente à adoção pela sociedade contemporânea de alguns dos valores estruturantes do modelo capitalista como a competição, o individualismo, a busca por vantagens e recompensas, a falta de envolvimento com os projetos coletivos e comunitários, a naturalização da desigualdade social e econômica.

Desse modo, a construção dos significados atribuídos a ideia de sustentabilidade, sobretudo na perspectiva do Sul - não referida no aspecto geográfico mas, conforme proposto por Boaventura de Souza Santos na discussão sobre Epistemologias do Sul (BARRETO, 2014), na proposição de outras formas de pensar o mundo e caminhos alternativos aos modelos hegemônicos impostos pelos países desenvolvidos - vem sendo fundada na ideia de reestruturação do modelo de sociedade vigente. Assim, de um lado observa-se uma perspectiva mais tecnológica e comportamentalista para a solução dos problemas ambientais, reconhecida por muitos autores como relacionada à ideia de educação para o desenvolvimento sustentável (GONZÁLEZ-GAUDIANO, 1998, 2005). De outro lado, busca-se na sustentabilidade socioambiental outro modelo de sociedade em que a participação, o envolvimento, o diálogo e o sentido de pertencimento comunitário, justiça e responsabilidade global sejam preponderantes nas decisões sobre um futuro comum a todos (LOUREIRO; LAYRARGUES, 2013).

No campo teórico da educação ambiental, estas posições são reconhecidas como tendências ou tipologias distintas, resumidas em pelo menos três principais: a conservacionista, a pragmática e a crítica (SILVA; CAMPINA, 2011; LOUREIRO; LAYRARGUES, 2013), sendo as duas primeiras mais identificadas com as concepções e práticas dos países desenvolvidos e a última relacionada aos países em desenvolvimento, sobretudo à tradição da educação ambiental na América Latina (GONZÁLEZ-GAUDIANO, 1998; LIMA, G., 2009). 
Sob o enfoque desse último caso, a universidade precisaria optar pela sustentabilidade, não para atender a um modismo ou para se sobressair diante das demais concorrentes em um mercado cada vez mais competitivo como é o do ensino superior, mas como forma de ser agente da transformação que se deseja para o planeta.

\section{Institucionalização da sustentabilidade nas universidades}

Vários estudos analisam os processos de transição das instituições de ensino superior em US, e indicam aspectos que favorecem e outros que dificultam esse que é necessariamente um processo que propõe mudanças profundas na forma de funcionamento da universidade. Dentre os fatores que dificultariam a implementação e a institucionalização da sustentabilidade nas universidades, destacam-se: i) as resistências pessoais às mudanças e à inovação (LOZANO, 2006; ĆULUM, 2014); ii) as barreiras institucionais e sistêmicas às mudanças (HARRIS; CRANE, 2002 apud PEREIRA; JABBOUR; DE OLIVEIRA; TEIXEIRA, 2013); iii) a percepção limitada do conceito de sustentabilidade por parte dos gestores (WRIGHT; HORST, 2013); iv) os problemas na condução do processo participativo na institucionalização da sustentabilidade (DISTERHEFT; CAEIRO; AZEITEIRO; LEAL FILHO, 2014). Por outro lado, há fatores que potencialmente promovem a implementação da sustentabilidade nas universidades, dentre os quais podem ser citados: i) a formalização do compromisso da instituição com a sustentabilidade (LOZANO; CELEUMANS; ALONSO-ALMEIDA; HUISINGH; LOZANO, F.; WAAS; LAMBRECHTS; LUKMAN; HUGÉ, 2015); ii) o compromisso dos gestores e líderes com o tema (BURFORD; HOOVER; VELASCO; JANOUŠKOVÁ; JIMENEZ; PIGGOT; PODGER; HARDER, 2013); iii) o estabelecimento de uma forma consistente de avaliar e relatar o desempenho institucional em termos da sustentabilidade (LOZANO, 2011; CELEUMANS; MOLDEREZ; VAN LIEDEKERKE, 2014; AMARAL; MARTINS; GOUVEIA, 2015); iv) a abordagem participativa na implementação da sustentabilidade (DISTERHEFT; CAEIRO; RAMOS; AZEITEIRO, 2012), incluindo o envolvimento dos professores e com atenção especial aos funcionários técnico-administrativos (BRINKHURST; ROSE; MAURICE; ACKERMAN, 2011); v) a disseminação do conhecimento sobre o processo e incentivos ao envolvimento de professores e técnicos (LEVY; MARANS, 2012); vi) a integração da sustentabilidade nos currículos em perspectiva crítica e complexa (SEGALÀS; MULDER; FERRER-BALAS, 2012).

Quanto aos processos de institucionalização da sustentabilidade nas universidades, as principais estratégias discutidas na literatura são a top-down e a participativa. Disterheft, 
Caeiro, Ramos e Azeiteiro (2012) analisaram universidades europeias que possuíam algum sistema de gestão ambiental, identificando os tipos de sistemas adotados e as formas de implementação e monitoramento, considerando distintos níveis de participação. A principal conclusão é a de que a perspectiva participativa parece ser mais abrangente do que a top-down porque não visa apenas a melhoria no desempenho ambiental da instituição, mas uma melhor incorporação da sustentabilidade em todos os níveis de atuação da universidade, sobretudo na formação dos estudantes para o enfrentamento da questão junto à sociedade.

Considerando que a mudança almejada se trata de um processo, alguns autores propõem estágios de implementação da sustentabilidade nas universidades. Leal Filho (2009) propõe três estágios, considerando a compreensão dos princípios da sustentabilidade e as ações concretas realizadas pela instituição. No estágio 1, os princípios não são integralmente compreendidos e não há esforços aparentes da instituição. No estágio 2 há significativas ações e os conceitos são amplamente compreendidos, havendo projetos de promoção da sustentabilidade em contextos específicos. No estágio 3 a universidade apresenta compromissos de longo prazo, tais como políticas, grupo de coordenação das ações de sustentabilidade e estabelecimento de formas de certificação de suas ações.

Gutiérrez-Barba e Martínez-Rodríguez (2010) também propõem três estágios, considerando o grau de maturidade da instituição em relação à sustentabilidade. Nessa proposta, a maturidade incipiente estaria relacionada ao uso de um tema que marque a opção pela sustentabilidade (como projetos sustentáveis, liderança em desenvolvimento sustentável, entre outros), no entanto com pouca estrutura administrativa e ações curriculares concretas. A maturidade intermediária inclui a distribuição de conteúdos em temas diversos como o entorno natural, economia, civismo e valores, com estruturas acadêmicas e organizativas para tais fins. As instituições ditas maduras seriam as que prescindem de espaços curriculares formais de conteúdo relativo à sustentabilidade, pois esta estaria presente no cotidiano. A partir da classificação proposta por Gutiérrez-Barba e Martínez-Rodríguez (2010), Martínez-Fernández e González Gaudiano (2015) sugerem que as instituições do ensino superior possam ser classificadas em de primeira geração, quando estão focadas principalmente em processos acadêmicos, e de segunda geração, quando se envolvem com a comunidade externa buscando impactar aspectos econômicos e sociais.

A partir da análise de experiências de universidades em diversas partes do mundo, Velazquez, Munguia, Platt e Taddei (2006) desenvolveram um modelo para a implementação de uma US em quatro fases, na seguinte sequência: $\left(1^{\mathrm{a}}\right)$ desenvolver uma visão de sustentabilidade para a universidade; $\left(2^{\mathrm{a}}\right)$ incluir a sustentabilidade dentre as missões da 
universidade; $\left(3^{\mathrm{a}}\right)$ criar um comitê de sustentabilidade para estabelecer políticas, objetivos e coordenar iniciativas; $\left(4^{\mathrm{a}}\right)$ implantar estratégias de sustentabilidade nas dimensões da educação, pesquisa, extensão e gestão do campus

A proposta apresentada no AISHE (Auditing Instrument for Sustainability in Higher Education), desenvolvido pela Fundação Europeia de Gestão da Qualidade, vai mais além, considerando cinco estágios de aprofundamento da sustentabilidade na instituição, partindo da existência de atividades individuais e pontuais (estágio1), passando por ações coletivas de curto prazo (estágio 2), ações sistêmicas de médio prazo com avaliação de metas pré-estabelecidas (estágio 3), ações de longo prazo com envolvimento de atores externos como escolas e avaliação comparativa com outras instituições (estágio 4), até ações de longo prazo com reflexo na sociedade de modo geral, com avaliação externa e repercussão de destaque dentre as demais instituições (estágio 5) (ROORDA, 2001).

O uso de sistemas de gestão ambiental (SGA) que incluam formas de monitorar, avaliar e relatar as ações de sustentabilidade tem sido apontado como essencial para apoiar a transição para as universidades sustentáveis (LOZANO, 2006; CELEUMANS; MOLDEREZ; VAN LIEDEKERKE, 2014). Amaral; Martins; Gouveia (2015) apresentam uma revisão sobre as principais formas que têm sido utilizadas para implementar, avaliar e relatar a sustentabilidade nas universidades, discutindo desde os tipos de sistemas de gestão ambiental mais genéricos e inicialmente adotados pelas universidades, tais como o padrão ISO 14001 e o EMAS (European Union Eco-Management and Audit Scheme), até aqueles desenvolvidos especialmente para o caso da sustentabilidade nas universidades, como o já citado AISHE, STARS (Sustainability Tracking, Assessment \& Rating System) e GASU (Graphical Assessment of Sustainability in Universities), que procuram incorporar a complexidade das dimensões e missões das universidades, sobretudo a educação. Dado que muitas universidades optam por criar seu próprio sistema de avaliar e relatar as suas ações de sustentabilidade, existem outros diversos modelos para a gestão e/ou avaliação da sustentabilidade, com diferentes enfoques. Recentemente, Disterheft, Caeiro, Leal Filho e Azeiteiro (2016) desenvolveram um modelo, chamado INDICARE, de apoio à avaliação dos processos participativos de implementação da sustentabilidade nas instituições de ensino superior. Segundo os autores, ao invés de apresentar mais uma ferramenta de controle das ações das universidades, a proposta visa oferecer aos participantes uma possibilidade de reflexão sobre a qualidade do processo participativo vivenciado na instituição, fortalecendo tanto seu potencial transformador, como o surgimento de novas visões de mundo e valores que aproximem indivíduos e natureza. 


\section{Proposição e questões de pesquisa}

Partimos do entendimento de que a sustentabilidade no Ensino Superior já não deve mais ser vista como uma "inovação radical", conforme sugerido por Lozano (2006) e outros, pois atualmente o tema está, de alguma forma, presente nas instituições dadas as demandas de diferentes origens, tanto pressões externas quanto internas, e iniciativas vindas tanto de indivíduos ou pequenos coletivos quanto da direção superior. Daí ser difícil classificar uma universidade como sendo ou não sustentável, ao passo que é possível considerar distintos estágios de institucionalização da sustentabilidade.

A forma como se dá o processo de institucionalização da sustentabilidade nas universidades, incluindo as dimensões e aspectos que recebem maior destaque, provavelmente está relacionada à percepção sobre o tema e o conceito de sustentabilidade adotado pelos sujeitos que coordenam o processo. Já o processo de institucionalização pode ser facilitado ou dificultado de acordo com as conjunturas estruturais e culturais da própria universidade, assim como com as estratégias de institucionalização assumidas.

Tomando o caso de uma universidade portuguesa, buscamos neste estudo responder às seguintes questões: Como a universidade vem agindo no sentido de sua transição para ser uma universidade sustentável? Quais as estratégias assumidas e as possíveis razões que as justificam? Quais as perspectivas para o processo de institucionalização da sustentabilidade nessa universidade?

A partir do caso dessa universidade, ampliamos o foco da discussão para os processos de institucionalização da sustentabilidade nas universidades de uma forma geral.

\section{A Universidade estudada}

A universidade em foco nesse estudo abriga cerca de 15 mil estudantes em mais de 80 cursos de graduação e pós-graduação. O número de docentes e investigadores é pouco maior que mil e há cerca de 650 funcionários não docentes. A universidade conta com três campi, mas o estudo foi realizado no campus principal, pela sua dimensão e representatividade face aos restantes, bem como pelo fato de comportar a totalidade da oferta de ensino universitário da instituição. Este campus tem uma área total maior que 70 mil metros quadrados, e cerca de 60 edifícios.

A universidade tem forte repercussão regional e também se sobressai no contexto nacional e internacional, situando-se em colocações de destaque dentre as universidades portuguesas nos rankings internacionais de avaliação do ensino superior. Trata-se de uma 
universidade relativamente recente, quer no panorama do ensino superior nacional, quer a nível europeu, que apresenta uma estrutura organizacional algo diferente das restantes universidades Portuguesas, notadamente a existência de uma estrutura matricial, departamental e sem faculdades, com uma governação muito centrada na reitoria.

A análise da estrutura de ensino e pesquisa revela uma tendência à promoção da interdisciplinaridade, como é o caso da estratégia de implementação de espaços interdisciplinares de pesquisa, chamados de Plataformas Tecnológicas, como também de cursos que agregam e mobilizam distintos setores da universidade.

Apesar de não haver nenhum centro ou departamento especificamente dedicado à sustentabilidade, a lista de cursos de mestrado e doutorado permitiu identificar ao menos onze deles com abordagem claramente voltada a aspectos da sustentabilidade, desde o campo dos estudos ambientais até a educação e a gestão, passando pela tecnologia aplicada a sustentabilidade. Não foi identificado se há integração entre tais cursos.

Há aproximadamente um ano da realização desse estudo, existe em atividade um grupo multidisciplinar de professores, designado pelo reitor para elaboração de um plano estratégico para a transição da universidade para uma universidade sustentável, referido nas seções seguintes como Grupo de Missão (GM).

\section{O Estudo de Caso}

Segundo Yin (1994), o estudo de caso é uma pesquisa empírica que investiga um fenômeno contemporâneo no seu contexto real, quando os limites entre o contexto e o fenômeno não são evidentes; é uma metodologia adequada a responder perguntas do tipo como e por que. De fato, a institucionalização da sustentabilidade nas universidades é um tema pouco analisado na literatura do ponto de vista processual, ou seja, das opções tomadas pela universidade na condução do processo e suas razões. A instituição escolhida para a pesquisa é considerada adequada para o estudo de caso por ser representativa de uma universidade de porte médio, com reconhecida qualificação no ensino e na pesquisa, e cujos órgãos de governação apresentam clara intencionalidade em priorizar a sustentabilidade como marca do campus.

A estrutura da pesquisa segue o design sugerido por Yin (1994), com o desenvolvimento de uma proposição teórica para guiar a coleta de dados e a triangulação dos dados a partir de múltiplas fontes de evidências. Assim, os dados foram construídos a partir da análise da literatura sobre a sustentabilidade no ensino superior, análises de documentos da instituição, observação do campus e entrevistas semiestruturadas com diferentes atores associados à 
governação e com poder de decisão quanto às questões relativas à sustentabilidade, bem como com outros atores não diretamente ligados à governação ou ao tema da pesquisa.

\subsection{Entrevistas}

Foram entrevistados dezoito membros da comunidade acadêmica, doze professores e seis funcionários, divididos em três grupos. O grupo 1 é composto por professores diretamente envolvidos com o tema, sendo um membro da direção superior da universidade, um professor de ciências sociais com atuação nas temáticas ambiental e comunitária, e cinco membros do já referido Grupo de Missão (GM), oriundos das áreas de ambiente, educação, economia, engenharia e design. O grupo 2 é composto por cinco professores de departamentos distintos e não diretamente relacionados à temática dessa investigação, e o grupo 3 por seis funcionários ligados à área administrativa com atuação em diferentes setores da universidade e que também não têm uma relação direta com a temática em estudo.

Foram realizadas entrevistas presenciais individuais com os professores do grupo 1, enquanto os grupos 2 e 3 participaram de entrevistas presenciais focais em duas sessões distintas. As entrevistas foram gravadas, transcritas, e submetidas à análise de conteúdo, de acordo com o proposto por Bogdan e Biklen (1994). Nas entrevistas individuais foram exploradas as concepções de US, as ações realizadas pela universidade no campo da sustentabilidade, e as características da universidade que favorecem e que dificultam que a transição da universidade para uma US. Nas entrevistas focais foram exploradas as concepções de US, as impressões sobre a atuação da universidade na promoção da sustentabilidade e as possíveis contribuições dos professores e funcionários ao processo. Após leituras sucessivas das entrevistas, as falas foram analisadas em dois temas: o conceito de US e a transição da universidade para US. No segundo tema, foi construída uma grelha a fim de quantificar a ocorrência de fatores que, segundo os entrevistados, favorecem essa transição ou que a dificultam. As categorias que emergiram das falas estão evidenciadas nas tabelas 1 e 2 .

\subsection{Análises de documentos}

Os principais documentos analisados foram os Estatutos da Universidade, a apresentação da missão institucional no website da universidade, o plano de ação apresentado por ocasião da candidatura do reitor, bem como os documentos gerados pelo GM, nomeadamente o plano estratégico e o plano de ação para 2015. Foram também analisadas notícias divulgadas no jornal online da universidade e nas edições da newsletter desse jornal, 
semanalmente distribuída por e-mail para toda comunidade acadêmica, publicadas no período entre janeiro e junho de 2015.

$\mathrm{Na}$ análise buscou-se a presença de elementos indicadores do conceito de US adotado pela universidade assim como as estratégias estabelecidas visando a transição da universidade para US.

\section{Concepções sobre a Universidade Sustentável}

A missão apresentada no website da universidade não apresenta referência clara à sustentabilidade, no entanto, os Estatutos da Universidade indicam que, para concretizar a sua missão, a Universidade deve promover o pensamento e a consciência crítica da sociedade, e define a universidade como uma instituição socialmente responsável e implicada no desenvolvimento sustentável.

A análise das notícias divulgadas no jornal da universidade sugere que a pesquisa produzida relacionada à sustentabilidade parece regular e consistente. De 32 notícias sobre a pesquisa produzida na universidade, no período analisado, 12 tinham relação direta com a sustentabilidade, e foram produzidas por distintos setores da universidade em diferentes vertentes do tema, tais como proteção e monitoramento da biodiversidade, nanotecnologia aplicada à reutilização de resíduos industriais, educação para o desenvolvimento sustentável, avaliação de impactos ambientais, mudanças climáticas, processos de descontaminação de águas e defesa do patrimônio urbano e natural.

Dentre as notícias de divulgação de eventos e cursos, presentes nas edições das newsletter analisadas, foram encontradas algumas ações relacionadas à sustentabilidade relativas à dimensão de educação (realização de oficinas), extensão (encontros relacionados a projetos comunitários) e vivências no campus (discussões e ações sobre mobilidade suave), notadamente em menor escala que na dimensão pesquisa.

Duas notícias trataram da dimensão gestão do campus. Uma se referia às soluções de sustentabilidade e de eficiência energética, associadas à arquitetura do campus, e a outra apresentava o Grupo de Missão para o Desenvolvimento Sustentável da Universidade, ao qual cabia elaborar uma proposta de estratégia para o campus sustentável da universidade, a partir de uma demanda do reitor.

Os dois documentos produzidos pelo GM revelam consistência com o contexto internacional (sobretudo as proposições da ONU) que imputa às Instituições de Ensino Superior (IES) responsabilidades em relação a construção de uma sociedade mais sustentável, e também 
uma compreensão da abordagem complexa e holística da sustentabilidade. É citado o compromisso estabelecido pelo reitor com o tema, que em seu programa de ação utiliza com frequência os termos 'campus exemplar', 'campus sustentável' e 'universidade cívica' para se referir à meta almejada para a universidade.

A estratégia proposta pelo GM deixa clara a opção por um processo cíclico de planejamento, monitorização e avaliação, e indica como áreas prioritárias a educação, a gestão dos campi, o envolvimento da comunidade e a investigação. Na educação são propostos programas internos de formação da comunidade acadêmica, o desenvolvimento de competências dos estudantes ligadas ao desenvolvimento sustentável e a participação da universidade no projeto Europeu "University Educators for Sustainable Development" (UE4SD). Na gestão dos campi são propostas ações de gestão de recursos, desmaterialização de processos administrativos, manutenção de estruturas, valorização da biodiversidade e definição de princípios reguladores da qualificação dos campi, visando à certificação da universidade nas normas ISO, relacionadas ao desenvolvimento sustentável. No envolvimento da comunidade são previstas ações como oficinas, atividades integradoras, debates e a participação da comunidade nos programas de monitorização da qualidade ambiental. Na investigação destaca-se a valorização do envolvimento dos estudantes, o foco no desenvolvimento sustentável dos próprios campi, a produção de projetos interdepartamentais e de impacto internacional, e a preocupação com a comunicação interna e externa dos resultados das investigações, incluindo a transferência tecnológica com o tecido industrial local. $\mathrm{Na}$ conclusão da apresentação das propostas é percebida a intencionalidade da universidade de se tornar referência no tema.

Considerando o ponto de partida para as ações, o documento estratégico produzido pelo GM afirma que: As componentes social, econômica e de governação são fundamentais para o sucesso desta ação, e serão sempre consideradas, no entanto as opções estratégicas para 20142020 , visam diretamente a componente ambiental, numa perspectiva contínua de co-benefício social e econômico.

O documento também reconhece que ações propostas poderão aumentar o grau de competitividade da universidade, e que este é um aspecto fundamental no programa Horizon 2020, da comunidade europeia.

O plano de ação indica que a ligação e o envolvimento doutros grupos da universidade, nomeadamente a Associação Académica, a Associação de Funcionários, os Núcleos de Estudantes, a Associação de Antigos Estudantes, potenciarão a adesão da comunidade ao Campus Sustentável. Nesse sentido, uma primeira ação do GM junto aos estudantes foi a 
organização da semana de recepção aos calouros que incluiu dois dias de atividades voltadas à sensibilização para o tema da sustentabilidade, com atividades no próprio campus e no centro da cidade.

Todos os entrevistados do grupo 1 apresentaram conhecimento teórico consistente associado a sua percepção sobre a US, com frequentes referências às dimensões ambiental, social e econômica, conforme expresso na fala desse representante do GM (Grupo 1): $O G M$ tem representantes de vários departamentos da universidade, desde o ambiente até a educação, passa pela comunicação e arte, mecânica, biologia, geociências, gestão, e só isso já mostra que a preocupação com a sustentabilidade na universidade tenta assentar nos pilares típicos da sustentabilidade, a componente ambiental, a componente social e a componente econômica.

Entre os docentes não diretamente relacionados à temática da sustentabilidade (Grupo 2), houve tanto opiniões que reforçaram uma perspectiva complexa da sustentabilidade, incluindo a responsabilidade social e rejeitando o uso de rankings de sustentabilidade para as universidades, como posicionamentos de desconforto em relação ao tema, pelo fato do mesmo ser percebido como fora da área de atuação desses professores. Nesse caso uma ideia defendida foi remeter o assunto ao grupo mais especializado no tema: Há pessoas que fazem investigação na área e dão formação na área e podem dar contributos em mais longo prazo, e é mais fácil envolver essas pessoas em algum tipo de prática, pois não exige sair do paradigma de suas tarefas (Docente - Grupo 2).

Os funcionários não docentes (Grupo 3) apresentaram uma visão de US voltada prioritariamente ao uso sustentável dos recursos energia e água, e descarte de resíduos, analisando diversas possibilidades de ações de promoção da sustentabilidade no âmbito da universidade, dentre elas a perspectiva política do processo: Pode-se ter edifícios novos, edifícios inteligentes, pessoas sensíveis, mas depois se não tiver uma política de sustentabilidade, não vale a pena, morre ali (Funcionário - Grupo 3).

De modo geral, nos três grupos foi dado destaque à necessidade de redução do impacto ambiental resultante do funcionamento da própria universidade e a sua correspondência com a redução de custos, levando, portanto, a uma necessidade de gestão dos recursos. O aspecto social foi explorado com menos detalhamento nas falas dos entrevistados. As responsabilidades da universidade relativas à sustentabilidade são consideradas similares a qualquer instituição, no entanto é reconhecido o papel intrínseco da componente educativa: É possível uma universidade sustentável se conseguirmos uma visão sistêmica do que se passa numa universidade e onde é que estão as áreas críticas, as áreas de desperdício, as áreas de ineficiência, e elas forem claramente identificadas e solucionadas, mas isso não tem sentido se 
não houver aqui uma forte componente de educação, de educação de todos os agentes envolvidos (Representante do GM - Grupo 1).

Referências a uma abordagem holística da sustentabilidade, incluindo outros elementos na discussão, são pouco frequentes, mas foram feitas por alguns dos entrevistados, sobretudo membros do GM, como nesse caso: Temos que considerar a sustentabilidade social, não é só uma questão de dinheiro, mas uma questão de bem-estar. Portanto, na sustentabilidade devese ter em conta em primeiro a sociedade e até o indivíduo e sua relação com a natureza, com a alimentação, com o lazer, etc., tudo isso faz parte de um pensamento equilibrado sobre o que é sustentabilidade e não apenas a otimização dos recursos (Representante do GM - Grupo 1).

\section{Fazendo a transição para a Universidade Sustentável}

Todos os entrevistados do Grupo 1 afirmaram que a transição para uma Universidade Sustentável é objetivo da universidade nesse atual momento, nomeadamente pela criação do GM. Argumentaram que a universidade já apresentava iniciativas pontuais, mas reconhecem que ainda há um percurso para a transição para a sustentabilidade: Eu acho que a universidade já colocou esse tema não só na agenda da sua política, das suas ações, mas já implementou algumas medidas. Agora se tu me perguntares se numa visão mais holística essa questão já está clarificada eu diria que a universidade começa a dar alguns passos, mas isso ainda não está suficientemente central, ainda não é visível pelo menos (Representante do GM - Grupo 1).

Vários dos elementos deste grupo de entrevistados fazem referência ao papel decisivo do compromisso do reitor no processo de transição para a sustentabilidade, sugerindo uma perspectiva top-down do processo, em que cabem ao reitor não apenas as primeiras ações para iniciar o processo e o apoio para sua continuidade, mas também o poder de decisão e de implementação das ações sugeridas, como nos seguintes depoimentos desses representantes do GM - Grupo 1: a) A sustentabilidade está presente no discurso do reitor, no programa de campanha e no plano estratégico. A criação do grupo de missão é um indicativo claro que a universidade pretende ser uma universidade sustentável; b) Há eventos e iniciativas que estão dispersas, e o fato de haver uma top-down, do próprio reitor ter criado um grupo de missão, se calhar ajuda a participar das atividades que já existem e promover outras, criando sinergias; c) Nós não somos um grupo executivo, nós somos um grupo que apresenta propostas ao reitor para aquilo que o reitor achar por bem implementar.

De outro lado, professores e funcionários não diretamente relacionados com a temática da sustentabilidade (os entrevistados dos grupos 2 e 3) revelaram desconhecer o GM ou, no 
caso de o conhecerem, afirmaram não ter informações sobre quem seriam seus membros e quais ações estariam desenvolvendo. Indicaram ainda que percebem a universidade pouco sensível ao tema da sustentabilidade, ainda que sejam recorrentes, em todos os grupos, as referências às iniciativas de implantação de painéis solares em alguns edifícios, do programa de incentivo ao uso de bicicletas e da construção de edifícios com aproveitamento da luz solar. Foi dito que ações como estas conferem visibilidade à instituição no setor, mas restaria dar o passo seguinte, que é a construção de estratégias para a institucionalização do tema. Uma professora relembra que o que é implementado é muito menos dos resultados das investigações que foram feitas na própria universidade sobre essa questão, enquanto uma funcionária conclui que o que há é uma consciência economicista, mas não necessariamente sustentável.

Dentre as características da universidade que possam favorecer sua transição para uma US (Tabela 1) foi dado destaque para a história da universidade e seu pioneirismo no estudo do ambiente e na relação com a região onde se situa: Essa foi a primeira universidade do país a ter formação em ambiente, portanto há um historial de preocupação da universidade com essa questão. Temos os cursos, a investigação, isso mostra que a universidade desde a sua raiz está preocupada com essas questões (Representante do GM - Grupo 1).

A proximidade espacial entre os diferentes departamentos e do campus com a cidade e a natureza local foram também qualidades destacadas. Apesar de não ter sido lembrada pelos demais grupos, a organização matricial da estrutura acadêmico-administrativa da universidade foi um fator bastante destacado pelos entrevistados do grupo 1, como no caso desse representante do GM: O campus foi desenhado desde o início para ser um campus, eu diria, fácil de estar num processo de sustentabilidade. A própria cultura organizacional da universidade tende a facilitar isso, mais matricial, mais horizontal, não tem corpos intermédios muito forte como faculdades, o que torna também a gestão mais facilitada para processos de mudanças, processos de adaptação, de introdução de instrumentos de sustentabilidade.

Tabela 1 - Características da universidade que podem favorecer a sua transição para universidade sustentável de acordo com os entrevistados, e o número de entrevistados que as citaram (num total de 18 entrevistados).

\begin{tabular}{lc}
\hline \multicolumn{1}{c}{ Características da Universidade } & Ocorrências \\
\hline As relações históricas da universidade com a região e a temática ambiental & 6 \\
A estrutura física do campus e a sua localização geográfica & 5 \\
A competência local sobre o tema & 4 \\
A organização matricial & 4 \\
A pré-disposição da comunidade acadêmica para colaborar na transição & 4 \\
As edificações modernas (em relação a outras instituições europeias) & 3 \\
O envolvimento em redes internacionais ligadas à sustentabilidade & 2 \\
O tamanho relativamente pequeno da universidade & 2 \\
Possibilidades de relações interdisciplinares & 1 \\
Opção estratégica clara pela sustentabilidade & 1 \\
\hline
\end{tabular}

Fonte: Autores. 
As referências aos aspectos que podem dificultar a transição para uma US variaram entre temas amplos, como a ausência de uma política de sustentabilidade, e temas mais específicos, como a ausência de ecopontos no campus necessários ao descarte adequado dos resíduos sólidos (Tabela 2). Houve também uma diferença qualitativa entre os grupos. Enquanto os entrevistados incluídos no Grupo 1 deram destaque para aspectos técnicos, como as limitações orçamentárias, equipamentos e procedimentos administrativos obsoletos do ponto de vista da sustentabilidade, os professores que constituíram o Grupo 2, e sobretudo os funcionários entrevistados (Grupo 3), chamaram a atenção para os aspectos humanos e comunitários da questão, destacando as dificuldades de integração e de diálogo, e as resistências pessoais às mudanças, como nos exemplos a seguir: a) $O$ mais difícil é sempre ultrapassar as limitações orçamentais, a dificuldade de intervir nos edifícios a tempo de poder promover as mudanças necessárias em termos de infraestrutura. Esse é o dilema habitual que qualquer universidade vive (Representante do GM - Grupo 1); b) Apesar de não sermos uma universidade muito grande e de estarmos perto uns dos outros, às vezes não sabemos bem o que os outros estão a fazer; podíamos trabalhar mais em conjunto, mas nem sempre isso acontece (Docente - Grupo 2); c) Me parece que tem algo aqui que é fundamental: que não há espaço de diálogo, e isto independentemente do assunto ser a sustentabilidade ou qualquer outro assunto \&\#091;...\&\#093; Se calhar, uma das políticas de sustentabilidade é pôr as pessoas a falar do assunto. Se calhar é o principal (Funcionária - Grupo 3).

A participação no processo foi um tema recorrente entre os professores e funcionários sem relação direta com o tema (grupos 2 e 3). Foi destacada a necessidade de haver feedback das sugestões que por vezes são dadas, a fim de evitar a desmotivação e reforçar o interesse na participação de todos os atores. Por outro lado, os funcionários pontuaram como obstáculo à participação a sua baixa representatividade nos espaços decisórios da universidade, e as reduzidas oportunidades de realizar debates sobre temas diversos, inclusive a sustentabilidade: É absolutamente essencial sentir-se parte da instituição, e sentir-se parte da instituição é meio caminho andado para se querer o melhor para ela e se conseguir das pessoas melhores atitudes (Funcionária - Grupo 3). Os professores, por sua vez, destacaram a grande quantidade e diversidade de cargos e funções que lhes são atribuídos, os quais dificultam a participação em ações como as relativas à sustentabilidade, que tendem a ser pouco valorizadas na carreira docente: Há muitas exigências a puxar para todos os lados e o tempo não é elástico, e as pessoas começam a ter que se focar no que é essencial. Então, acho que essas questões dos 
incentivos são mais importantes para impulsionar o envolvimento das pessoas (Docente Grupo 2).

Tabela 2 - Características da universidade que podem dificultar a sua transição para uma universidade sustentável de acordo com os entrevistados, e o número de entrevistados que as citaram (num total de 18 entrevistados).

\begin{tabular}{lc}
\hline \multicolumn{1}{c}{ Características da Universidade } & Ocorrências \\
\hline Falta de integração e diálogo & 6 \\
Resistências pessoais às mudanças & 3 \\
Equipamentos obsoletos do ponto de vista da sustentabilidade & 2 \\
Limitações orçamentárias & 2 \\
Carga de trabalho elevada e falta de incentivos & 2 \\
Ausência de ecopontos para o descarte adequado dos resíduos & 2 \\
Resistências do sistema tradicional de gestão de documentos & 1 \\
Manutenção e intervenções em um campus relativamente grande & 1 \\
Baixa aplicação interna dos conhecimentos produzidos pela universidade & 1 \\
Ausência de uma política de sustentabilidade & 1 \\
Ausência da preocupação com a sustentabilidade nos contratos públicos & 1 \\
\hline
\end{tabular}

Fonte: Autores.

\section{Discussão}

A universidade estudada atua nas dimensões da sustentabilidade sugeridas na literatura, com ações evidentes nos campos da investigação, ensino e extensão. Os documentos formais da instituição trazem algumas referências à sustentabilidade, mas percebe-se que o processo de institucionalização do tema encontra-se em fase inicial, o que justificaria a classificação da instituição nos estágios de maturidade incipiente ou intermediário, propostos por GutiérrezBarba e Martínez-Rodríguez (2010) e na primeira geração, conforme Martínez-Fernández e González Gaudiano (2015).

Tomando como referência os estágios de sustentabilidade propostos por Leal Filho (2009), a universidade em questão pode ser enquadrada no estágio 2 encaminhando-se para o 3 , restando para isso a formalização institucional das políticas de sustentabilidade, ora situadas no contexto de um mandato de um reitor, e o estabelecimento de instrumentos de avaliação, ora em fase de elaboração. Já de acordo com os estágios propostos no AISHE, a universidade estudada pode ser enquadrada no estágio 3, apresentando metas e ações ainda relacionadas ao contexto interno da instituição com vistas ao médio prazo (2014-2020), no entanto indicando que pretende se tornar uma referência no setor, e atingir o estágio 5, no qual as ações de sustentabilidade da universidade se repercutem na sociedade. Mesmo sem considerar o tema da 
sustentabilidade, diversos autores têm destacado a necessidade das universidades aumentarem seu envolvimento com a sociedade, assumindo seu papel de instituição indutora de desenvolvimento econômico e social, e promotora de mudança social e cultural (SANTOS; ALMEIDA FILHO, 2012; PEDROSA, 2014). No entanto, o sucesso dessa ação demanda a criação de novas configurações institucionais (MELLO, 2011), o que inclui a formalização de canais de diálogo da universidade com atores locais e regionais, incluindo escolas, empresas, instituições de pesquisa, gestão pública, organizações não-governamentais e movimentos sociais.

Considerando as fases de institucionalização da sustentabilidade propostas por Velazquez, Munguia, Platt e Taddei (2006), a universidade parece contemplar parcialmente as etapas 2, 3 e 4, mas sobretudo a primeira, que é a construção de uma visão de sustentabilidade para a universidade, pode não estar sendo suficientemente explorada. Os membros do GM apresentaram percepções fundamentadas para um conceito de US, sobretudo com base nas orientações gerais da ONU para o desenvolvimento sustentável. De fato, a perspectiva crítica ao modelo de desenvolvimento econômico não é observada nas falas dos entrevistados, levando à ausência de referências claras aos aspectos políticos da sustentabilidade, seguindo a tendência observada para os países desenvolvidos, sobretudo no continente europeu. Adicionalmente, como uma visão mais detalhada e consensual não consta de documentos formais, por exemplo, dentre as missões da universidade ou em documentos específicos relativos à US, a comunidade acadêmica poderá ter dificuldades na compreensão do tema e até no estabelecimento de critérios para a priorização de ações de sustentabilidade a serem implementadas. Por exemplo, dentre os aspectos culturais relativos à sustentabilidade, pode-se considerar incoerente que em uma US ocorram atividades agressivas e moralmente reprováveis entre estudantes, como ocorrem na recepção dos novos alunos (nas ditas praxes académicas ou trotes aos calouros) na quase totalidade das universidades portuguesas e brasileiras, incluindo a universidade estudada. Nesse sentido, concorda-se com González-Gaudiano, Meira-Cartea e Martínez-Fernández (2015), quando afirmam que é difícil pensar que as universidades possam servir como modelo gerador de mudanças sociais profundas da relação com a biosfera se os estudantes continuarem a serem formados a partir dos mesmos conhecimentos, práticas culturais e modelos de produção ambientalmente insustentáveis e socialmente injustos que nos levaram a situação atual. Em um raciocínio similar, um dos professores sem ligação direta à temática em análise considerou que uma universidade qualquer que tenha cuidados com as áreas verdes e seja referência na gestão energética e de água, mas que não se preocupe com as condições de trabalho e a remuneração dos funcionários, não poderia ser considerada sustentável. Assim, e como foi sugerido pelos 
funcionários entrevistados (Grupo 3), o processo de discussão sobre o tema pode ser fundamental para permitir a reflexão sobre a complexidade da sustentabilidade, o envolvimento comunitário a longo prazo, o levantamento de ações já existentes e a inspiração para ações com maior possibilidade de virem a ser implementadas com sucesso.

Como já pontuado, a perspectiva política da sustentabilidade esteve pouco presente nos discursos e documentos analisados. Essa dimensão, no entanto, parece ser estratégica na transição para as sociedades sustentáveis (e, em consequência, para as US), pois inclui, dentre outros aspectos, o pensamento crítico sobre os modelos de organização da sociedade, e a participação na tomada de decisões como método prático de ensinar e aprender sobre democracia. A educação para a decisão e para a responsabilidade social e política (FREIRE, 1967; LIMA, L., 2002; 2014) converge para o que se espera da dimensão educativa nas universidades sustentáveis (SEGALÀS; MULDER; FERRER-BALAS, 2012; DISTERHEFT; CAEIRO; AZEITEIRO; LEAL FILHO, 2014; SOUZA, 2016). Nesse sentido, cabe reforçar o que vem sendo defendido em outros trabalhos a respeito da necessidade da universidade buscar sua própria transformação para poder contribuir verdadeiramente para a construção das sociedades sustentáveis (DISTERHEFT; CAEIRO; AZEITEIRO; LEAL FILHO, 2014; BIZERRIL; ROSA; CARVALHO; PEDROSA, 2015). Esse processo de reflexão e realização de ações concretas requer tempo e envolvimento da comunidade acadêmica, que precisa ser sensibilizada para o fato de que a sustentabilidade diz respeito a todos os habitantes do planeta e não apenas a um grupo específico de estudiosos dessa temática. Também é preciso criar formas de reconhecimento da participação nesse processo (como na proposta de DISTERHEFT; CAEIRO; LEAL FILHO; AZEITEIRO, 2016), revisando os atuais indicadores de produção do trabalho de professores e funcionários, e a própria concepção do que sejam os papéis das universidades na sociedade e no marco da nova ordem mundial. Desse modo, conforme González-Gaudiano, Meira-Cartea e Martínez-Fernández (2015, p. 71), "é necessário insistir na função da universidade como o laboratório da realidade atual dentro das condições concretas da região no contexto global".

Relativamente à utilização de rankings para aferir a sustentabilidade nas universidades, os professores sem relação direta com a temática (Grupo 2) consideraram-na contraproducente, sugerindo que essa forma de avaliação tende a forçar uma uniformização das universidades, e que a US pode ser desviada da proposta original se for reduzida a uma forma de captação de financiamentos, estratégia de marketing ou de ampliação do potencial competitivo. A respeito disso, González-Gaudiano, Meira-Cartea e Martínez-Fernández (2015, p. 84) alertam que a escolha dos indicadores de sustentabilidade podem converter um processo que deve ser 
necessariamente cooperativo e solidário - valores fundamentais em uma cultura profunda de sustentabilidade - em um mecanismo competitivo de luta pela captação de recursos públicos e privados.

A forma de implementação top-down, identificada na universidade estudada, traz, conforme Disterheft, Caeiro, Ramos e Azeiteiro (2012), vantagens e desvantagens. Se, por um lado, o apoio da gestão superior é essencial para o sucesso do processo e possibilita inúmeras facilidades às ações pretendidas, também é verdade que cria o desafio de envolver um maior número de pessoas da comunidade no processo, nomeadamente professores, funcionários e estudantes, e também a comunidade externa associada à universidade. A dependência excessiva do reitor para o andamento do processo aumenta as chances dos esforços se perderem na transição para um outro grupo que assuma a gestão da instituição e não dê sequência às ações iniciadas. Nos processos de envolvimento e de promoção da participação vale considerar que os compromissos gerados são maiores dentre aqueles que participam no planejamento e concepção da proposta do que dentre aqueles que apenas aderem às proposições.

A análise dos fatores facilitadores e limitadores do processo de transição para uma US indica que, apesar de haverem barreiras a vencer, tal como destacado em outros estudos como, por exemplo, as restrições orçamentárias (WRIGHT; HORST, 2013; MARTÍNEZFERNÁNDEZ; GONZÁLEZ-GAUDIANO, 2015), as características específicas de cada universidade devem ser consideradas de forma que os processos de institucionalização da sustentabilidade se deem em tempos e condições distintas, de acordo com as condições disponíveis em cada caso. Na instituição estudada, foi interessante notar o papel desempenhado pela estrutura acadêmico-administrativa, nomeadamente a estrutura matricial, e suas consequências nas relações interpessoais dos professores, o que parece impactar positivamente o trabalho que o GM vem desenvolvendo, sendo este um fato também observado para outras universidades (BIZERRIL, 2013). Merece destaque também a consciência de que os conhecimentos e competências necessárias para realizar essa transição, na maioria das vezes, estão presentes na própria universidade e o desafio passa por mobilizá-los no sentido do trabalho coletivo e interdisciplinar. De outro lado, os funcionários técnico-administrativos afirmaram encontrar dificuldades em efetivar seu envolvimento com o tema em decorrência da baixa representatividade nos espaços decisórios e da ausência de canais formais de diálogo entre si e a administração superior. Mais uma vez vem a tona o papel da organização política e da gestão participativa na universidade como elemento central para viabilizar a US. 


\section{Considerações finais}

O caso da universidade estudada pode ser representativo de diversas dentre as milhares de universidades existentes que podem ou estão a trilhar o necessário percurso rumo a uma US. Na conjuntura atual da Educação Superior, em que a tendência é de privilegiar a universidade empreendedora em relação à universidade cívica (LIMA, 2012; ĆULUM, 2014), a gestão orçamentária e a definição de prioridades de atuação da universidade que transcendam o tripé ensino-pesquisa-extensão surgem como fatores determinantes para a implantação da US.

Nesse cenário, parece-nos que a sustentabilidade na universidade precisa ser estimulada, pensada e realizada a partir das condições e características de cada instituição, e não meramente aferida ou quantificada para fins de avaliação e comparação. Em outras palavras, a opção da universidade pela sustentabilidade deve figurar dentre as formas de exercer sua autonomia como resposta às demandas sociais da atualidade, e não no simples atendimento de regras préestabelecidas por estruturas reguladoras da qualidade do ensino superior.

Apesar dos instrumentos existentes de avaliação da sustentabilidade nas universidades indicarem possibilidades diversas, nos parece sensato que as ações tomadas sejam escolhidas e agregadas à rotina da universidade de modo gradativo, a partir do aumento do compromisso da instituição e da comunidade acadêmica, e de um processo continuado de reflexão e busca por superação das contradições entre o discurso da US e as práticas realizadas na universidade. A convicção da administração superior em considerar a sustentabilidade como missão da universidade é essencial no processo, contudo não garante a sua efetividade, que necessariamente requer a reflexão e a transformação da comunidade acadêmica de um modo amplo. A criação de um fórum permanente de sustentabilidade - com representações dos órgãos de governança, professores, funcionários e estudantes - associado a estruturas de participação mais abertas que permitam a qualquer membro da comunidade encaminhar sugestões, que sejam consideradas e respondidas, pode apoiar o início do processo. No entanto, deve-se ter como horizonte a incorporação da sustentabilidade às práticas cotidianas para que estruturas específicas para esse fim se tornem desnecessárias, conforme sugerido por Gutiérrez-Barba e Martínez-Rodríguez (2010) no caso das universidades maduras.

Sugerem-se quatro ações estratégicas para as universidades que buscam a transição para US: (i) promover internamente ampla discussão visando a adoção de uma perspectiva holística de US e buscando a coerência nas suas ações cotidianas; (ii) buscar a institucionalização do tema por meio de documentos oficiais da universidade, considerando a diversidade interna e as 
especificidades de cada universidade; (iii) valorizar a gestão democrática e participativa como forma de possibilitar o envolvimento, a continuidade das ações a longo prazo e o processo reflexivo e formativo das pessoas para além do espaço universitário; (iv) criar canais formais de integração com a sociedade localmente, incluindo suas diversas representações, como forma de ampliar o potencial de ação da universidade local e regionalmente, e fortalecê-la como modelo gerador de mudanças em direção às sociedades sustentáveis.

\section{Agradecimentos}

Agradecemos ao apoio da Universidade de Brasília, Universidade de Aveiro e CIPES (Centro de Investigação de Políticas do Ensino Superior) para o desenvolvimento desta pesquisa de pós-doutoramento do primeiro autor, e a cada um dos entrevistados por sua disponibilidade e pela rica oportunidade de troca de experiências.

\section{Referências}

ALSHUWAIKHAT, H. M.; ABUBAKAR, I. An integrated approach to achieving campus sustainability: assessment of the current campus environmental management practices. Journal of Cleaner Production, v. 16, p. 1777-1785, 2008.

AMARAL, L. P.; MARTINS, N.; GOUVEIA, J. B. Quest for a sustainable university: a review. International Journal of Sustainability in Higher Education, West Yorkshire, v. 16, n. 2, p. 155-172, 2015.

BARRETO, J. M. Epistemologies of the South and Human Rights: Santos and the Quest for Global and Cognitive Justice. Indiana Journal of Global Legal Studies, Bloomington, v. 21, n. 2, p. 395-422, 2014.

BIZERRIL, Marcelo X. A. A estrutura acadêmica do campus da Universidade de Brasília em Planaltina-DF e seu potencial para a promoção do trabalho interdisciplinar. In:

CONFERÊNCIA DA FORGES - Política e gestão da Educação Superior nos países e regiões de língua portuguesa, 3. 2013, Lisboa. Atas... Lisboa: Universidade de Lisboa, 2013. p. 1-11.

BIZERRIL, Marcelo; ROSA, Maria João; CARVALHO, Teresa; PEDROSA, Júlio. A sustentabilidade socioambiental no ensino superior: um tema integrador para os países de língua portuguesa? Revista da FORGES, Lisboa, v. 2, n. 2, p. 99-115, 2015.

BOGDAN, Robert; BIKLEN, Sari. Investigação qualitativa em educação: uma introdução à teoria e aos métodos. Porto: Porto Editora, 1994.

BRINKHURST, M.; ROSE, P.; MAURICE, G.; ACKERMAN, J. D. Achieving campus sustainability: top-down, bottom-up, or neither? International Journal of Sustainability in Higher Education, West Yorkshire, v. 12, n. 4, p. 338-354, 2011.

BURFORD, G.; HOOVER, E.; VELASCO, I.; JANOUŠKOVÁ, S.; JIMENEZ, A.; PIGGOT, G.; PODGER, D.; HARDER, M. K. Bringing the "Missing Pillar" into Sustainable

Development Goals: Towards Intersubjective Values-Based Indicators. Sustainability, Basel, v. 5, p. 3035-3059, 2013. 
CELEUMANS, Kim; MOLDEREZ, I.; VAN LIEDEKERKE, L. Sustainability reporting in higher education: a comprehensive review of the recent literature and paths for further research. Journal of Cleaner Production, v. 106, 2014.

CORTESE, Anthony D. The critical role of higher education in creating a sustainable future. Planning for Higher Education, Ann Arbor, v. 31, n. 3, p. 15-22, 2003.

ĆULUM, B. Croatian academics and university civic mission integration: possibilities and constraints. In: BRANKOVIĆ, J.; KLEMENČIĆ, M.; LAŽETIĆ, P; ZGAGA, P. (Eds.). Global challenges, local responses in higher education: the contemporary issues in national and comparative perspective. Rotterdam: Sense Publishers, 2014. p. 59-78.

DISTERHEFT, Antje; CAEIRO, S. S. F. S.; RAMOS, M. R.; AZEITEIRO, U. M. M. Environmental Management Systems (EMS) implementation processes and practices in European higher education institutions - Top-down versus participatory approaches. Journal of Cleaner Production, v. 31, p. 80-90, 2012.

DISTERHEFT, Antje; CAEIRO, S.; AZEITEIRO, U.M.; LEAL FILHO, W. Sustainable universities - a study of critical success factors for participatory approaches. Journal of Cleaner Production, v. 106, n. 1, p. 11-21, 2014.

DISTERHEFT, Antje; CAEIRO, S.; LEAL FILHO, W.; AZEITEIRO, U. M. The INDICARE-model - measuring and caring about participation in higher education's sustainability assessment. Ecological Indicators, v. 63, p. 172-186, 2016.

FREIRE, Paulo. Educação como prática da liberdade. Rio de Janeiro: Paz e Terra, 1967.

GONZÁLEZ-GAUDIANO, Edgar. The Latin-American perspective on the debate on education for sustainability. Environmental Communicator, v. 28, n. 5, p. 11-12, 1998.

GONZÁLEZ-GAUDIANO, Edgar. Education for Sustainable Development: configuration and meaning. Policy Futures in Education, v. 3, n. 3, p. 243-250, 2005.

GONZÁLEZ-GAUDIANO, Edgar; SILVA-RIVERA, E. Education: A Road to Nowhere or a Path for a More Sustainable Future? A Southern Perspective. In: SELBY, D., KAGAWA, F. (Eds.). Sustainability frontiers: critical and transformative voices from the borderlands of sustainability education. Toronto: Barbara Budrich Publishers, 2015. p. 43-57.

GONZÁLEZ-GAUDIANO, Edgar; MEIRA-CARTEA, P. A.; MARTÍNEZ-FERNÁNDEZ, C. N. Sustentabilidad y universidad: retos, ritos y posibles rutas. Revista de la Educación Superior, México, v. 44, n. 175, p. 69-93, 2015.

GUTIÉRREZ-BARBA, B. E.; MARTÍNEZ-RODRÍGUEZ, M. C. El plan de acción para el desarollo sustentable en las instituciones de educacíon superior: escenarios posibles. Revista de la Educación Superior, México, v. 2, n. 154, p. 111-132, 2010.

HARRIS, L. C.; CRANE, A. The greening of organizational culture: management views on the depth, degree and diffusion change. Journal of Organizational Change Management, v. 15, n. 3, p. 214-234, 2002.

KARATZOGLOU, B. An in-depth literature review of the evolving roles and contributions of universities to education for sustainable development. Journal of Cleaner Production, v. 49, p. 44-53, 2013.

LEAL FILHO, Walter. Sustainability at universities - opportunities, challenges and trends.. Frankfurt: Peter Lang, 2009. v. 31 
LEVY, B. L. M.; MARANS, R. W. Towards a campus culture of environmental sustainability. International Journal of Sustainability in Higher Education, West Yorkshire, v. 13, n. 4, p. 365- 377, 2012.

LIMA, Gustavo F. C. Educação ambiental crítica: do socioambientalismo às sociedades sustentáveis. Educação e Pesquisa, São Paulo, v. 35, n. 1, p. 145-163, 2009.

LIMA, Licínio C. Organização escolar e democracia radical: Paulo Freire e a governação democrática da escola pública. São Paulo: Cortez, 2002.

LIMA, Licínio C. A gestão democrática das escolas: do autogoverno à ascensão de uma pósdemocracia gestionária? Educação \& Sociedade, Campinas, v. 35, n. 129, p. 1067-1083, 2014.

LOUREIRO, Carlos F. B.; LAYRARGUES, P. P. Ecologia política, justiça e educação ambiental crítica: perspectivas de aliança contra-hegemônica. Trabalho, Educação e Saúde, Rio de Janeiro, v. 11, n. 1, p. 53-71, 2013.

LOZANO, Rodrigo. Incorporation and institutionalization of SD into universities: breaking through barriers to change. Journal of Cleaner Production, v. 14, p. 787-796, 2006.

LOZANO, Rodrigo. The state of sustainability reporting in universities. International Journal of Sustainability in Higher Education, West Yorkshire, v. 12, n. 1, p. 67-78, 2011.

LOZANO, Rodrigo; CELEUMANS, Kim; ALONSO-ALMEIDA, M.; HUISINGH, D.; LOZANO, F. J.; WAAS, T.; LAMBRECHTS, W.; LUKMAN, R.; HUGÉ, J. A review of commitment and implementation of sustainable development in higher education: results from a worldwide survey. Journal of Cleaner Production, v. 108, p. 1-18, 2015.

MARTÍNEZ-FERNÁNDEZ, Cynthia N.; GONZÁLEZ-GAUDIANO, E. J. Las políticas para la sustentabilidad de las instituciones de educación superior en México: entre el debate y la acción. Revista de la Educación Superior, Mexico, v. 2, n. 174, p. 61-74, 2015.

MELLO, Alex F. Globalização, sociedade do conhecimento e educação superior: os sinais de Bolonha e os desafios do Brasil e da América Latina. Brasília: Editora UnB, 2001.

PEDROSA, Julio. Autonomy and Accountability in University Governance. In: BERGAN, S.; EGRONPOLAK, E.; KOHLER, J.; PURSER, L. (Eds.). Leadership and governance in higher education. Berlin: Raabe Academic Publishers, 2014. p. 23-39.

PEREIRA, G. S. M.; JABBOUR, C.; DE OLIVEIRA, S. V. W. B.; TEIXEIRA, A. A. Greening the campus of a Brazilian university: cultural challenges. International Journal of Sustainability in Higher Education, West Yorkshire, v. 15, n. 1, p. 34-47, 2014.

ROORDA, N. Auditing instrument for sustainability. In: CORCORAN, Peter B.; WALS, Arjen E. (Eds.). Higher education and the challenge of sustainability. Dutch: Dutch Foundation on Sustainable Higher Education; Dutch Ministry of Environmental Affairs, 2001.

SANTOS, Fernando S.; ALMEIDA FILHO, N. A quarta missão da Universidade: internacionalização na sociedade do conhecimento. Brasília: Editora UnB; Coimbra: Imprensa da Universidade de Coimbra, 2012.

SEGALÀS, J.; MULDER, K.F.; FERRER-BALAS, D. What do EESD "experts" think sustainability is? Which pedagogy is suitable to learn it? Results from interviews and Cmaps analysis gathered at EESD 2008. International Journal of Sustainability in Higher Education, West Yorkshire, v. 13, n. 3, p. 293-304, 2012. 
SILVA, Rosana L. F.; CAMPINA, Nilva N. Concepções de educação ambiental na mídia e em práticas escolares: contribuições de uma tipologia. Pesquisa em Educação Ambiental, Rio Claro, v. 6, n. 1, p. 29-46, 2011.

SOUZA, V. M. Para o mercado ou para a cidadania? A educação ambiental nas instituições públicas de ensino superior no Brasil. Revista Brasileira de Educação, Rio de Janeiro, v. 21, n. 64, p. 121-142, 2016.

VELAZQUEZ, Luís; MUNGUIA, N.; PLATT, A.; TADDEI, J. Sustainable university: what can be the matter? Journal of Cleaner Production, v.14, p.810-819, 2006.

WRIGHT, T.; HORST, N. Exploring the ambiguity: what faculty leaders really think of sustainability in higher education. International Journal of Sustainability in Higher Education, West Yorkshire, v.14, n.2, p. 209 - 227, 2013.

YIN, R.K. Case study research: design and methods. New York: Sage Publications, 1994.

Marcelo Ximenes Aguiar Bizerril

${ }^{1}$ Universidade de Brasília

Planaltina | DF | Brasil. Contato: bizerril@unb.br

ORCID https://orcid.org/0000-0002-2993-155X

Maria João Rosa

${ }^{2}$ Universidade de Aveiro | Departamento de Economia, Gestão, Engenharia Industrial e Turismo Aveiro | Portugal. Contato: m.joao@ua.pt

ORCIID (iD) https://orcid.org/0000-0003-2956-6801

Teresa Carvalho

${ }^{3}$ Universidade de Aveiro | Departamento de Ciências Sociais, Políticas e do Território Aveiro | Portugal. Contato: teresa.carvalho@ua.pt

ORCIID (iD https://orcid.org/0000-0002-3368-3990 